\title{
Review Article: Rapid review of hand hygiene efficacy in reduction catheter associated urinary tract infections
}

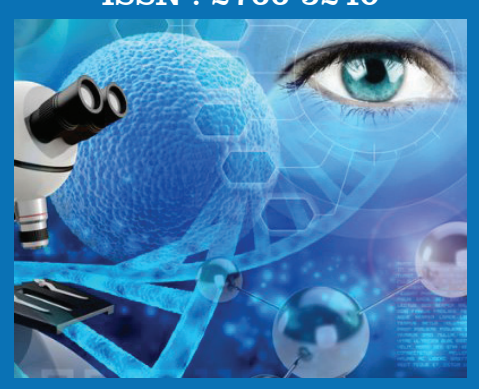

Issue Type: Volume 2 Issue 1
Author Name:

Muath Elwaisi ${ }^{1}$, Dr. Mahmoud Al-

Masaeed $^{2}$, Rawan Alsababha ${ }^{3}$,

Dr. Muhammad Alqudah ${ }^{4}$

${ }^{1}$ Registered Nurses, Master of Nursing from Nursing and Midwifery school, University of Wollongong, Australia.

${ }^{2}$ Researcher, $\mathrm{RN}$ and $\mathrm{PhD}$ from College of Health, Medicine and Wellbeing, University of Newcastle, Australia, and Faculty of Medicine and Health Sciences, Universiti Putra Malaysia, under Jointly Awarded Doctoral Degree (JADD)

${ }^{3}$ Researcher, RN, MSN and a PhD candidate at School of Nursing \& Midwifery, Western Sydney University

${ }^{4} \mathrm{RN}, \mathrm{MSN}, \mathrm{PHD}$ and Lecturer at School of Nursing and Midwifery, Faculty of Health and Medicine, University of Newcastle

Corresponding Author:

Dr. Mahmoud Al-Masaeed

Citation: Dr. Mahmoud Al-Masaeed, Rapid review of hand hygiene efficacy in reduction catheter associated urinary tract infections

Received Date: 12th Dec 2021

\section{Published Date: 28th Dec 2021}

Copyrights: Dr. Mahmoud Al-Masaeed This is an open access article distributed under the Creative Commons Attribution License, which permits unrestricted use, distribution, and reproduction in any medium, provided the original work is properly cited.

\section{ABSTRACT}

Background: This review has been undertaken to evaluate the effectiveness of the hand hygiene in the prevention of catheter associated urinary tract infections among patients admitted in the acute care settings. Catheter associated infections are totally preventable and the morbidity and mortality associated with these infections cause a burden on the healthcare system and the economy of the country. Hand hygiene of the health care personnel's is the most effective and economical way in the prevention of infections associated with the urinary catheters.

Objective: This review to find out the effectiveness of hand hygiene for the prevention or reduction in the rate of catheter associated urinary tract infections among patients admitted in the acute care setting.

Result: Five different experimental studies identified the effectiveness of hand hygiene of healthcare workers on the rates of catheter associated urinary tract infections.

Conclusion: Hand hygiene is effective in the prevention of catheter associated urinary tract infections when health care workers perform it as per the five moments of hand hygiene given by WHO.

Keywords: Catheter associated urinary tract infections (CAUTI), hand hygiene, patients, acute care setting (ICU, critical care unit, surgical unit etc).

\section{Background}

Catheter associated urinary tract infections are the leading cause of hospital acquired infections in health care setting which is caused by the use of indwelling urinary catheters. Though, urinary tract infections are counted as fourth most common type of hospital acquired infections with a number of $40 \%$ reported in acute care settings (Elpern, 2016), but $80 \%$ of all these UTI's are caused by indwelling catheters (Boev \& Kiss, 2017). Indwelling urinary catheters are used in clients with special medical needs (Justus et al., 2016). It is commonly used in hospitals in critically ill patients, in postoperative patients, patients with poly trauma who require prolong immobilisation and in other indications for the correct measurement of the urine output (Al-Hameed et al., 2018). These patients have multiple insults to their normal defence mechanisms; therefore, they are at high risk of developing the infection when urinary catheter is used (Galiczewski, 2016). The first and the most important intervention in the care of urinary catheter to prevent CAUTI is hand hygiene (Gupta et al., 2018).

Catheter associated urinary tract infections are defined by the Centers for Disease Control and Prevention in 2013 as "an infection caused by the insertion of an indwelling urinary catheter for more than 2 calendar days on the day of event where placement of the device is considered to be day one" (cited in Justus et al., 2016). The presence of indwelling catheter can increase the patient's risk for infection by three to seven percent (Peter et al., 2018). The rates of CAUTI in a healthcare setting are calculated as "the number of CAUTIs divided by catheter days multiplied by 1,000" (McCalla et al., 2018).

CDC given a criteria for CAUTI for its evaluation in a patient. According to this criteria, firstly the patient should have the indwelling catheter in place from two days or removed just a day before, secondly, patient should show the symptoms such as fever, suprapubic tenderness, costovertebral angle pain or tenderness, 
urinary urgency, dysuria and lastly, the urine culture of the patient is identified with two species of organisms with one bacterium of 105 colony forming units per millilitre. Therefore, it is evident that CAUTI increases the usage of antimicrobials with the prolongation in hospital stay of patients. This will also increase the the hospital expenses associated with it (Peter et al., 2018). Thus, the mortality and morbidity rates associated with it also increased and these will further increase the cost associated with it (Boev \& Kiss, 2017).

Despite taking all the preventive measures the incidence of CAUTI increased by $6 \%$ from year 2009 to 2013 when the goal was set to decrease the infection rate by $25 \%$ in United States (Sopirala et al., 2018). But in United States the incidence of CAUTI for adult inpatient floors ranged from 0.1 to 3.1, 1.2 to 5.3 per 1000 catheter days in adult critical care units as per the data reported to the CDC National Healthcare Safety Network (Hollenbeak \& Schilling, 2018). In Australian heath care context, indwelling catheters are used in almost $26 \%$ of patients admitted in acute care settings and $1 \%$ of these patients got affected by CAUTI (Mitchell et al., 2012). The cost of treating these infections calculated to be approximately $\$ 1000$ which includes the cost of diagnostic tests and medications used. Therefore, burden on the health system and economy is quite evident (Hollenbeak \& Schilling, 2018).

However, all these expenditures are prevented by preventing CAUTI, which will also show improvement in the quality of health care (Peter et al., 2018). CDC and world health organisation support hand hygiene in the prevention of catheter associated infections as it is the most simple, efficient and economical way of preventing the transmission of pathogens in healthcare setting. CDC defines hand hygiene as "the practice of cleaning hands to prevent disease causing germs". But low compliance of healthcare workers to hand hygiene is often observed (McCalla et al., 2018). Many factors are observed for having the low compliance of healthcare workers towards handwashing. Some of these factors are: understaffing, overcrowding, belonging to certain professional category such as doctors, nursing assistants, working in areas such as intensive care units, lack of proper equipment's and infrastructure for hand washing (Allegranzi \& Pittet, 2009).

As hand hygiene is the foremost strategy to prevent infections, WHO adopt new global guidelines for effective hand hygiene. Under these guidelines WHO states 5 moments of hand washing which include the two moments of before and after. Hand hygiene Australia defines these critical moments as: before touching a patient: Moment 1, before a procedure (Moment 2), after a procedure (Moment 3 ), after touching a patient (Moment 4), and after touching a patient's surroundings (Moment 5). This will help in preventing the hospital acquired infections such as CAUTI and reduce the related effects of these infections on patient health outcome and on economic burden (White et al., 2015).

Despite using strategies to control the infection, preventable CAUTI remains problematic. Elpern in 2016 showed that there was an increase of $6 \%$ in the rate of catheter associated urinary tract infections despite using preventive strategies. The present literature review clearly identifies the evidence-based practice for CAUTI prevention but did not clearly state the importance of the hand hygiene as the integral component of this prevention strategy. Evidences showed that the compliance of health care workers to hand hygiene when doing the procedures have a significant effect on the incidence of CAUTI (Chih-
Cheng Lai et al., 2017). Previous research also identified lack of documentation regarding the importance of hand hygiene in all the procedures related to urinary catheters. This quality improvement project was based on the initiatives to improve the hand hygiene compliance among health care workers to bring the sustainable changes to prevent CAUTI among patients admitted in the acute care settings. This paper will discuss the effectiveness of hand hygiene in the prevention of CAUTI and compare the CAUTI rates before and after this intervention, thereby providing valuable recommendations to the researches and practitioners from its findings.

\section{Objective:}

The purpose of this rapid review is to identify, analyse and summarize the effectiveness of hand hygiene for the prevention or reduction in the rate of catheter associated urinary tract infections among patients admitted in the acute care setting. This review will be guided by the question: Is hand hygiene effective in the prevention of catheter-associated urinary tract infection in patients admitted in the acute care setting?

\section{Criteria for considering the studies: \\ Types of studies:}

The studies consist of existing experimental study designs with the pre-test and post-test interventional study design which a focus on hand hygiene intervention in the prevention catheter associated urinary tract infections. Most of the studies are primary studies.

\section{Types of participants:}

This review consists of studies with the title of patients admitted in acute care settings in any wards as patients are at high risk of developing infections associated with catheters due to the multiple insults to their normal defence mechanisms (Galiczewski, 2016) and the title of nurse or health care worker whose scope of practice involve direct patient care having catheters. These have direct contact with patient and their improper compliance to hand hygiene become major source of pathogens transmission, that will cause infection in patients (Al Kuwaiti, 2017). These healthcare workers involve the Registered nurses, Enrolled nurses and Assistance in nursing.

\section{Types of interventions:}

Studies with the intervention of hand hygiene used by the health care workers to increase patient safety by decreasing the chances of catheter associated urinary tract infections in acute care setting are included in this review (Al-Tawfiq et al., 2018).

\section{Control:}

The hand hygiene was considered as a control. WHO approaches to improve hand hygiene among health care workers are considered which show a significant difference in the rates of CAUTI with the application of this single simple intervention (Al Kuwaiti, 2017).

\section{Types of outcome measures:}

The main outcome of interest was the incidence and numbers of catheter associated urinary tract infections. Outcomes discussed the rate of CAUTI before hand washing and after maintaining proper hand hygiene. The rates were calculated as the numbers of CAUTI's divided by catheter days multiplied by 1000 . The studies which indicate the results before and after undertaking 
the hand hygiene as an intervention to prevent CAUTI were considered. Hand hygiene compliance rate of health care workers were also included as the secondary outcome. Compliance for hand hygiene was checked on two indications, first one was on entering the patient's room and the second one was after exiting the patient's room. Hand hygiene compliance were calculated as the number of hand hygiene events divided by the number of hand hygiene opportunities (McCalla et al., 2018).

\section{Search strategy:}

The data search strategy went through two phases. The relevant studies published within 10 years interval between 2009 to 2021 were selected so as to obtain the most recent evidences for ensuring the effectiveness of intervention. The databases used for research were MEDLINE plus and PUBMED.

\section{Phase 1:}

The initial search was conducted by the keywords taken from the abstract of the different research articles. Again, second search was conducted with all relevant keywords related to the topic of study. The advance search for the studies were done by limiting the articles to the English language, full articles and reports.

\section{Phase 2:}

For making the result more comparable, accurate and precise, the search was done by considering the inclusion and exclusion criteria. Papers were excluded which mention systematic reviews in their title. All the primary studies, reviews of reviews, interventions carried out in elderly in chronic care are excluded. Paper focusing on other interventions than hand hygiene was excluded. Papers focusing on children and the articles whose outcome did not include CAUTI rates were excluded. All the titles and abstracts were generated by the search were reviewed by the reviewer to identify the relevant articles. An evaluation of full texts was then conducted to determine if they met eligibility criteria. Search strategy provided in Appendix A, B and C.

\section{Methods of Review:}

Assessment of methodological quality:

Methodological quality of five different non-randomised articles were assessed by using Mixed Method Appraisal Tool (MMAT). According to Hong et al., (2018) Mixed method appraisal tool is used to assess the quality of mixed studies. The quality of the studies is assessed under five criterions given in this tool. Each criterion has five questions which was used to assess the quality according to study. The qualitative study, quantitative randomised control trial, quantitative non-randomised, quantitative descriptive and mixed method only papers rate under the five criteria in each category. The response of cannot tell depicts that the paper does not provide accurate information for the answer of 'yes' and 'no' to the question. This means that the paper needs further details to be reviewed.

\section{Data extraction and synthesis:}

The data was extracted by the single person. Data related to the study design, intervention of hand hygiene, compliance of health care workers to hand hygiene, rate of catheter associated urinary tract infections and result after this intervention were extracted. References were done manually. The details about the included studies were presented through a structured table format and then interpreted. The findings of repeated studies among the chosen studies were carefully synthesized. Data synthesis was done in a narrative and descriptive form.

\section{Review results: \\ Description of studies:}

Total 186 articles were retrieved by using two databases. A total number of 127 articles were retrieved from the database MEDLINE and 59 articles from PUBMED. Number of duplicates were removed. Papers retrieved for detailed examination were 149 and 132 articles were removed further after reviewing the title and abstract. 17 articles were reviewed further, and 11 articles are removed for not meeting the inclusion criteria. Full articles retrieved for further examination were 6 . Out of these 6 articles, one article was removed due to can't tell score of the MMAT, which means that article need further detailed review. Finally, five articles meeting the methodological quality were selected for inclusion.

Figure 1: Flow diagram of the paper selection process:

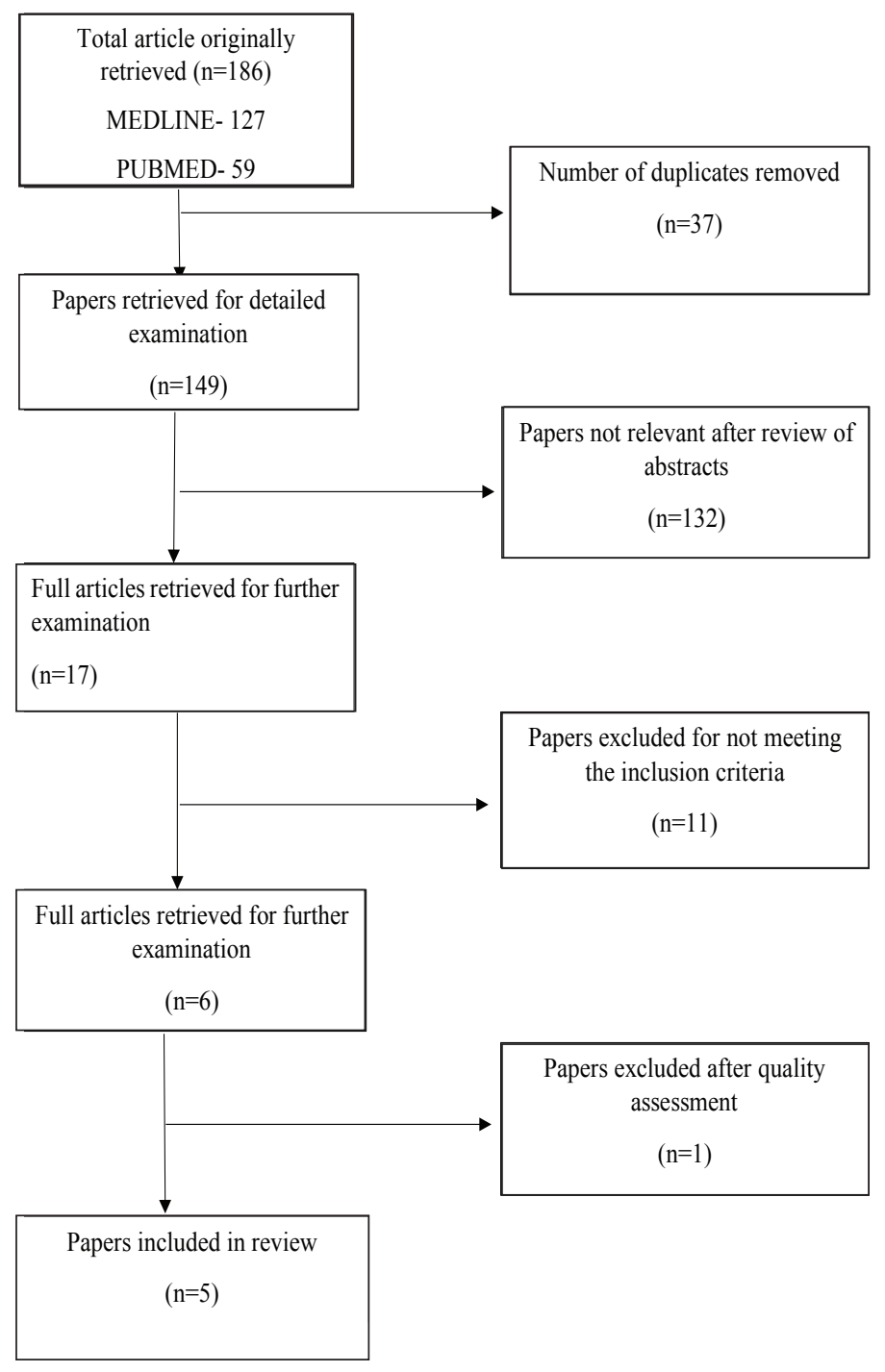

Among the five-existing different experimental, non-randomised studies which includes pre intervention and post intervention of hand hygiene, selected for review to check the effectiveness of hand hygiene in the prevention of catheter associated urinary tract infection. The study conducted by Al Kuwaiti (2017) is a prospective interventional study, study conducted by McCalla et al., (2018) is a non-randomised pre and post interventional study, 
study conducted by McCalla et al., (2017) is a retrospective cohort design and study by Al-Tawfiq et al., (2018) and Kachare et al., (2014) is a pre test and post test interventional study. All the studies are focused on hand hygiene as intervention. Study conducted by Kachare et al., focused on hand hygiene in the bundle of care. Both studies conducted by McCalla and study by Al Kuwaiti focused on compliance to hand hygiene by healthcare workers. All the studies were conducted in the different units of community hospitals such as surgical, oncology unit except the study by Al Kuwaiti which was done in a university hospital. The participants were the caregivers or healthcare professionals working in those units where the study was conducted in the given time frame. The time period of the studies conducted varies according to the study design. The study conducted by $\mathrm{Al}$ Kuwaiti (2017) had conducted for two years from 2014 to 2016 in the same hospital to check the effectiveness of intervention. In this study, during the pre-test, the compliance rate of hand hygiene for health care workers was increased by taking positive interventions such as providing education and training on this practice of hand hygiene, presenting visual displays to hand hygiene, increasing availability of alcohol-based hand rubs and water supply. The adherence to hand hygiene was gradually increased in this study from $50.17 \% \pm 9.00 \%$ in 2014 to $66.08 \%$ $\pm 7.43 \%$ in 2015 and $71.75 \% \pm 2.18 \%$ in 2016 and the rates of CAUTI were gradually decreased from $3.73 \pm 2.41$ in 2014 to $2.21 \pm 2.93$ in 2015 and $1.75 \pm 1.70$ in 2016 .

Additionally, the study conducted by McCalla et al., (2018) has 14,279 participants in pre intervention phase and 36, 890 participants in intervention period and this study was conducted from January 2014 to September 2017. The study focused on the hand hygiene opportunities and the increase in these opportunities with the application of automated system. The opportunities for handwashing were 60,167 in the month of march in 2015 when it was implemented but then increased to 696,928 between March 2016 to September 2017. Similarly, the CAUTI rate was 2.20 per 1,000 catheter days during preintervention period which was then decreased to 1.21 per 1,000 during the intervention period. This study represents $45 \%$ relative risk reduction in CAUTI by this implication.

The other study conducted by McCalla et al., (2017) focused on the educational strategies to increase the compliance of hand hygiene for healthcare workers in the same setting after noticing the rate of hand hygiene compliance in ICU unit, which was affected by the use of gowns and gloves. This study was conducted in two steps. Human observers were also used in the first phase and then in second phase automated hand hygiene compliance system was used. Compliance rate of healthcare workers to hand hygiene was recorded $98.8 \%$ in ICU when assessed by human observers and CAUTI rate was $1.5 \%$ in 2014. But hand hygiene compliance was $95.2 \%$ when assessed by automated system in 2015 and CAUTI rate was 1.7 in ICU. The $\mathrm{p}$ value was .03 at this time and incidence rate for CAUTI was 1.02 .

The study conducted by Al-Tawfiq et al., (2018) showed the different compliance rate of hand hygiene for daytime shifts and night-time shits which was $73.9 \%$ and $83.9 \%$ at baseline for day-time shifts and $92 \cdot 4 \%$ and $87 \cdot 3 \%$ during the intervention phase. He used the tool for improving hand hygiene by Joint Commission Centre for Transforming Healthcare's Web-based targeted solutions tool $\AA$ (TST $®)$. The data was collected according to the 5 moments of hand washing and the major reasons for being non-compliant to hand washing were detected as hands full of supply $27 \cdot 5 \%$, use of gloves $27 \cdot 2 \%$ and hands full of medications $23 \cdot 4 \%$. Other than this, frequent entry exit, follow entry/ exit were also involved. Education was provided to the groups as well who were non-compliant to hand washing to increase the compliance. The decrease in CAUTI rate was also observed from $5 \cdot 941$ to 0 per 1000 device days.

The last study performed by Kachare et al., (2014) in a medical centre. This study included intervention of hand hygiene along with bundle of care. Indications for catheter use were clearly given. The data was collected about the total patient's, patient's days, device days and number of CAUTI'S. the total number of patients included in the study was 2843 and a significant decrease in monthly patient days was observed which was recorded as $720.3 \pm 62.95$ versus $785.0 \pm 75.72$. the mean of CAUTI was observed 4.9 in preintervention and 0.9 in post intervention while the $\mathrm{p}$ value was 0.03 . on the other side the percentage of hand hygiene compliance of healthcare workers was recorded to be $58.3 \%$ in preintervention period and $91.7 \%$ in postintervention period.

Three studies were conducted in two-year time period while two of them completed in one-year time period. All studies aimed to decrease the rates of catheter associated urinary tract infections by increasing the compliance of healthcare workers to hand hygiene. All five studies showed the involvement of healthcare workers in bringing the change in their practice to reduce rate of CAUTI. some studies such as McCalla et al., also included other health care infections along with CAUTI.

\section{Results:}

\section{Quality of experimental studies:}

Five studies of high quality included for the rapid review through MMAT. All the five studies meet quantitative non-randomised criteria. The MMAT items like the confounders were missing in some studies. The total number of articles reviewed were 186. All the studies included were conducted in the acute care settings. The overall results of studies were analysed and extracted in a tabular form.

Effectiveness of proper hand hygiene by following the WHO five moments of hand washing were identified from the studies. In a study conducted by McCalla et al., (2017), effectiveness of the educational strategies was identified to improve the compliance of healthcare workers towards handwashing so that the catheter associated urinary tract infections can be reduced. Similarly, the study conducted by Al Kuwaiti (2017) identifies the strategies such as training, visual presentation of hand hygiene practice to improve compliance of healthcare workers to hand washing. These studies showed a positive effect in the reduction of catheter associated urinary tract infections with the increase in practice of hand hygiene among healthcare workers.

Another study conducted by McCalla et al., (2018) identified the effectiveness of automated hand hygiene compliance system to increase practice of hand hygiene among practitioners. But Kachare et al., (2014) concluded that the hand hygiene is more effective in the prevention of infections related to catheter if practised wit the bundle of care. In all the studies the effectiveness of hand hygiene was clearly stated by the infection rates before and after the intervention.

\section{Discussion:}

\section{Summary of findings:}

There are wide range of studies conducted on the role of hand hygiene in the reduction of catheter associated urinary tract infections among the patients admitted in the acute care settings. This rapid review has described the effectiveness of the hand 
hygiene in the prevention of CAUTI. This rapid review has extracted few strategies to increase the hand hygiene compliance among health care workers. These strategies involve education, training and involvement of automated hand hygiene compliance system in order to increase the healthcare worker's compliance of hand hygiene. Along with the strategies, the reasons of healthcare workers for being not compliant to hand washing are also identified. Some of these reasons explained are hands full of medications, frequent entry or exit and hands full of supply. After reviewing the effectiveness of hand hygiene, most of the studies showed that when the healthcare workers followed five moments of hand hygiene properly then the reduction in the rate of catheter associated urinary tract infections was evident among the admitted patients having urinary catheters in the units of hospital. Hence, hand hygiene is found effective in the prevention of CAUTI.

\section{Quality of evidence:}

The use of MMAT undertaken as a tool for assessing the methodological quality of the studies. This tool has helped in solving the challenges faced in assessing the quality of different experimental studies. Five experimental studies have been included with all the yes answers to the questions which shows the quality of the studies.

\section{Potential biases in the review:}

The review has addressed the effectiveness of hand hygiene in a comprehensive manner contributing to the present information in the prevention of catheter associated urinary tract infections, however there are number of limitations identified. Time period limitation of studies from 2009 to 2021 has limited the inclusion of research studies before 2009 which can explain in detail about the extent of CAUTI and the hand hygiene compliance of healthcare workers. This review consists the population of only adult people which will make difficult to interpret the effectiveness of hand hygiene in the children. The studies included in the reviews have not interpreted results according to the age group or gender, which may alter the association of hand hygiene compliance to the infection rate. Additionally, studies included were conducted in the small number of bed units, which might create difference in results.

\section{Conclusion:}

The effectiveness of hand hygiene which includes hand washing or hand disinfection was identified in the prevention of infections related to urinary catheter, when properly followed by the healthcare workers. All the studies identified the relation of hand hygiene compliance of healthcare workers to the catheter associated infections. The more compliant healthcare workers to hand hygiene by following five moments, the less chances of catheter associated urinary tract infections are there. This review also presents the recommendations to the practitioners and researches considering the findings from the existing studies:

\section{Recommendations to practitioners:}

- It is recommended to apply intervention on a large area to get more accurate results for the intervention.

- It is recommended to apply hand hygiene in association with bundle of care to prevent CAUTI.

- It is recommended to involve each member of healthcare team to decrease the infection rate because in some studies physicians are excluded from observations and experiments.

\section{Recommendations to researchers:}

- Children also need to be included in the studies to get more accurate results about the effectiveness of $\mathrm{HH}$ on different age groups.

- The effectiveness of automated hand hygiene compliance system in relation to the infection control should need to be evaluated further for its cost effectiveness and its extent in prevention of infections.

- The interventions should be applied to large area to get more precise results.

\section{References:}

Allegranzi, B., \& Pittet, D. (2009). Role of hand hygiene in healthcare-associated infection prevention. Journal of Hospital Infection, 73(4), 305-315. https://doi-org.ezproxy.uow.edu. au/10.1016/j.jhin.2009.04.019

Al-Hameed, F. M., Ahmed, G. R., AlSaedi, A. A., Bhutta, M. J., Al-Hameed, F. F., \& AlShamrani, M. M. (2018). Applying preventive measures leading to significant reduction of catheterassociated urinary tract infections in adult intensive care unit. Saudi Medical Journal, 39(1), 97-102. https://doi-org.ezproxy. uow.edu.au/10.15537/smj.2018.1.20999

Al Kuwaiti, A. (2017). Impact of a multicomponent hand hygiene intervention strategy in reducing infection rates at a university hospital in Saudi Arabia. Interventional Medicine \& Applied Science, 9(3), 137-143. https://doi-org.ezproxy.uow. edu.au/10.1556/1646.9.2017.24

Al-Tawfiq, J. A., Treble, M., Abdrabalnabi, R., Okeahialam, C., Khazindar, S., \& Myers, S. (2018). Using targeted solution tools as an initiative to improve hand hygiene: challenges and lessons learned. Epidemiology And Infection, 146(2), 276-282. https:// doi-org.ezproxy.uow.edu.au/10.1017/S0950268817002758.

Arabia. Indian Journal of Critical Care Medicine, 22(12), 858-861. https://doi-org.ezproxy.uow.edu.au/10.4103/ijccm. IJCCM_270_18

Boev, C., \& Kiss, E. (2017). Hospital-Acquired Infections: Current Trends and Prevention. Critical Care Nursing Clinics of North America, 29(1), 51-65. https://doi-org.ezproxy.uow.edu. au/10.1016/j.cnc.2016.09.012

Chih-Cheng Lai, Chun-Ming Lee, Hsiu-Tzy Chiang, ChingTzu Hung, Ying-Chun Chen, Li-Hsiang Su, ... Po-Ren Hsueh. (2017). Implementation of a national bundle care program to reduce catheter-associated urinary tract infection in highrisk units of hospitals in Taiwan. Journal of Microbiology, Immunology and Infection, (4), 464. https://doi-org.ezproxy. uow.edu.au/10.1016/j.jmii.2017.01.006

Elpern, E. (2016). Prevention of Catheter-Associated Urinary Tract Infections in Adults. Critical Care Nurse, 36(4), e9-e11. https://doi-org.ezproxy.uow.edu.au/10.4037/ccn2016208

Galiczewski, J. M. (2016). Interventions for the prevention of catheter associated urinary tract infections in intensive care units: An integrative review. Intensive and Critical Care Nursing, 32, 1-11. https://doi-org.ezproxy.uow.edu.au/10.1016/j. iccn.2015.08.007

Gupta, S. , Al Khaleefah, F., Al Harbi, I., Ahmed, F., Jabar, S., Torre, M., \& Mathias, S. (2018). An intervention study for the prevention and control of health care-associated infection in the critical cares area of a tertiary care hospital in Saudi

Hollenbeak, C. S., \& Schilling, A. L. (2018). The attributable cost of catheter-associated urinary tract infections in the United States: A systematic review. American Journal of Infection Control, 46(7), 751-757. https://doi-org.ezproxy.uow.edu. au/10.1016/j.ajic.2018.01.015 
Justus, T., Wilfong, D. N., \& Daniel, L. (2016). An Innovative Educational Approach to Reducing Catheter-Associated Urinary Tract Infections: A Case Study. Journal of Continuing Education in Nursing, 47(10), 473-476. https://doi-org.ezproxy.uow.edu. $\mathrm{au} / 10.3928 / 00220124-20160920-09$

Kachare, S. D., Sanders, C., Myatt, K., Fitzgerald, T. L., \& Zervos, E. E. (2014). Toward eliminating catheter-associated urinary tract infections in an academic health center. The Journal of Surgical Research, 192(2), 280-285. https://doi-org.ezproxy. uow.edu.au/10.1016/j.jss.2014.07.045

Krishna Institute of Medical Sciences University, 7(1), 55-66. Retrieved from https://search-ebscohost-com.ezproxy.uow. edu.au/login.aspx?direct $=$ true $\& \mathrm{db}=$ edselc $\& A N=$ edselc.2-52.0$85040916648 \&$ site $=$ eds-live

McCalla, S., Reilly, M., Thomas, R., McSpedon-Rai, D., McMahon, L. A., \& Palumbo, M. (2018). An automated hand hygiene compliance system is associated with decreased rates of health care-associated infections. American Journal Of Infection Control, 46(12), 1381-1386. https://doi-org.ezproxy.uow.edu. au/10.1016/j.ajic.2018.05.017

Mitchell, B. G., Fasugba, O., Gardner, A., Koerner, J., Collignon,
P., Cheng, A. C., ... Gregory, V. (2017). Reducing catheterassociated urinary tract infections in hospitals: study protocol for a multi-site randomised controlled study. BMJ Open, 7(11), e018871. https://doi-org.ezproxy.uow.edu.au/10.1136/ bmjopen-2017-018871

Peter, S., Devi, E. S., \& Nayak, S. G. (2018). Effectiveness of clinical practice guidelines on prevention of catheter-associated urinary tract infections in selected hospitals. Journal of

Sopirala, M. M., Syed, A., Jandarov, R., \& Lewis, M. (2018). Impact of a change in surveillance definition on performance assessment of a catheter-associated urinary tract infection prevention program at a tertiary care medical center. American Journal of Infection Control, 46(7), 743-746. https://doi-org. ezproxy.uow.edu.au/10.1016/j.ajic.2018.01.019

White, K. M., Jimmieson, N. L., Obst, P. L., Graves, N., Barnett, A., Cockshaw, W., ... Paterson, D. (2015). Using a theory of planned behaviour framework to explore hand hygiene beliefs at the "5 critical moments" among Australian hospital-based nurses. BMC Health Services Research, 15(1), 1-9. https://doiorg.ezproxy.uow.edu.au/10.1186/s12913-015-0718-2 